\title{
0steopathic manipulative treatment improves function and relieves pain in knee osteoarthritis: A single-blind, randomized-controlled trial
}

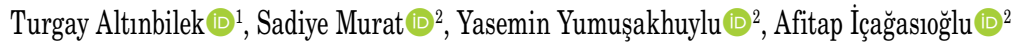 \\ ${ }^{1}$ Department of Physiotherapy and Rehabilitation, İstanbul Kültür University, Faculty of Health Sciences, İstanbul, Turkey \\ ${ }^{2}$ Department of Physical Medicine and Rehabilitation, İstanbul Medeniyet University, Göztepe Training and Research Hospital, İstanbul, Turkey \\ Received: February 07, 2017 Accepted: August 11, 2017 Published online: March 09, 2018
}

\begin{abstract}
Objectives: In this study, we aimed to compare the efficacy of osteopathic manipulative treatment (OMT) to exercise treatment in the knee osteoarthritis (OA).

Patients and methods: A total of 100 patients ( 9 males, 76 females; mean age $54.8 \pm 8.5$ years; range, 40 to 70 years) with Stage II-III bilateral knee OA enrolled to the study and randomized into two groups between January 2015 and June 2015. Group 1 performed exercise and received OMT and Group 2 performed exercise alone. We assessed the clinical parameters with Western Ontario MacMaster Questionnaire (WOMAC) pain score, WOMAC joint stiffness score, WOMAC physical function score, Visual Analog Scale (VAS) and 50-m walking time. All patients were assessed at the beginning of the study, just after the treatment, and four weeks after the treatment.

Results: There was no significant difference between groups in terms of physical examination and clinical assessment parameters before treatment. Functional improvement $(\mathrm{p}<0.05)$ and pain relief $(\mathrm{p}<0.05)$ were significantly higher in the exercise + OMT group.

Conclusion: Based on our study results, OMT is a particular treatment used by osteopathic physicians to complement conventional treatment of OA of the knee. In addition to the conservative treatment, OMT can be used.
\end{abstract}

Keywords: Exercise; function; gonarthrosis; manipulation; osteoarthritis; pain.

Osteoarthritis (OA) of the knee is a chronic, progressive and debilitating disease with noticeable pain and stiffness and causes physical disability. This condition also affects the patient's functionality. ${ }^{[1]}$ The management of the $\mathrm{OA}$ aims reducing pain, increasing physical function, preventing disability, and increasing quality of life. ${ }^{[2]}$ Depending on the stage of the disease, there are different treatment options. For patients who have signs and symptoms of early knee OA with generalized knee pain, conservative treatment methods should be chosen as first-line treatment. ${ }^{[3]}$

Osteopathic manipulative treatment (OMT) is a component of conservative care. Pathophysiology of the knee OA is partly related to changes in the autonomic nervous system, blood and lymph flow, fascial tension, range of motion limitations, and relationships of the length and tension of the muscles around the knee. ${ }^{[4,5]}$ Understanding of the somatic, sympathetic, and lymphatic systems and their integrated parts is necessary for understanding the etiology and management of knee pain. Therefore, evaluation of the surrounding muscle strength, flexibility, tone, skin topography, range of motion, and soft tissues is essential to effectively manage knee OA. Although lack of evidence, OMT has been advised as a complementary alternative therapy for patients with early stages of knee OA. ${ }^{[6,7]}$ Due to the insufficiency of larger clinical studies and strong evidence for the effectiveness of OMT for OA of the knee, we decided to design a study. In the present study, we aimed to assess the efficacy of OMT with exercise treatment in relieving the pain caused by OA of the knee.

Corresponding author: Yasemin Yumuşakhuylu, MD. İstanbul Medeniyet Üniversitesi Göztepe Eğitim ve Araştırma Hastanesi Fiziksel Tıp ve Rehabilitasyon Kliniği, 34722 Göztepe, Kadıköy, İstanbul, Turkey. e-mail: yassure@yahoo.com 


\section{PATIENTS AND METHODS}

A total of 100 patients ( 9 males, 76 females; mean age $54.8 \pm 8.5$ years; range, 40 to 70 years) who presented to the Physical Medicine and Rehabilitation outpatient clinics of İstanbul Medeniyet University, Göztepe Training and Research Hospital with knee pain lasting for more than six months were included in the study between January 2015 and June 2015. Six patients in the exercise + OMT group and nine patients in the exercise group were left the study by their own will. The study was completed with 85 patients. Patients diagnosed as bilateral primary knee $\mathrm{OA}$ according to the American College of Rheumatology (ACR) criteria. The anteroposterior (AP) and lateral knee radiographs taken to stage OA according to the Kellgren and Lawrence radiological staging scale and Stages II-III were included in the study. A written informed consent was obtained from each participant. The study protocol was approved by İstanbul Medeniyet University, Göztepe Training and Research Hospital Ethics Committee (Date: 30/12/2014; No: 0179). The study was conducted in accordance with the principles of the Declaration of Helsinki. Exclusion criteria were follows: inflammatory arthritis, soft tissue rheumatism an inflammation in the knee joint, higher erythrocyte sedimentation rate (ESR) and C-reactive protein (CRP), history of knee surgery, trauma (meniscopathy or instability), intraarticular intervention or physical therapy within the last six months. Also, patients using anti-inflammatory drugs other than simple analgesics, those using knee braces, patients with vascular and cardiovascular disease, paresis or neuropathy, intraarticular neoplasm, osteonecrosis and mental mood disorder and those with knee contracture were excluded from the study.

We evaluated the patients with a detailed medical history, and systemic and locomotor system examinations. All patients were questioned for age, sex, educational level, employment status, Body Mass Index, habits, complaint duration, use of analgesics, previous treatments, accompanying chronic disease, drugs used and previous surgical interventions.

Complete blood count, full urinalysis, ESR, CRP, fasting blood sugar, uric acid, kidney and liver function tests were analyzed in all patients. Radiological evaluation was performed with the AP and lateral radiographs according to the Kellgren and Lawrence radiological staging. ${ }^{[8]}$ On physical examination, the patients were evaluated in terms of pain, heat increase, effusion, crepitation, patellar grind test, valgus stress, varus stress, McMurray, anterior and posterior drawer test and Apley compression and distraction tests. Knee circumference and range of motion of all patients were actively measured using a standard goniometer. Clinical parameters were evaluated with the Western Ontario MacMaster Questionnaire (WOMAC), ${ }^{[9]}$ pain score (0-25), WOMAC stiffness of the joints score (0-10), WOMAC physical functioning of the joints score (0-85), Visual Analog Scale (VAS) and 50-m walking time.

The patients were prevented from taking nonsteroidal anti-inflammatory drugs one week before beginning of the study and during the study period. They were allowed to take paracetamol up to $3 \mathrm{~g}$ daily for pain control. The drugs they used due to systemic diseases were continued. The patients who met the criteria specified in the study protocol were randomized on www.randomizer.org ${ }^{[10]}$ website and divided into two groups. Each group consisted of 50 patients. Exercise + OMT were administered to the Group 1, while exercises alone were given to the Group 2.

An exercise program including quadriceps isometric strengthening straight leg lifting, iliotibial band, hamstring stretching, strengthening abductor and adductor muscle of the hip and stretching exercises was applied as 10-repetitive 3 set, two days a week, totally four sessions, in the clinic, and the program was taught to the patients for applying two times a day at home.

Adherence of the patients to the house exercise program was followed-up by weekly phone visits. Patients in the Group 1 were administered OMT as 3 min mobilization and $3 \mathrm{~min}$ compression for bilateral patellofemoral and tibiofemoral joint respectively with one minute intervals in addition to the exercise program. Following these applications, two min bilateral lower extremity pumping technique was performed. All these applications were taught to the patient for applying twice a day at home. Adherence of the patients to the more program was followed by weekly phone visits.

To provide standardization, all evaluations were made before treatment, after treatment and four weeks after treatment by a physiatrist who was blind to the treatment method. The exercises were applied and taught to the patients by another physiatrist. The OMT was performed by a third physiatrist who has been completed osteopathy training.

\section{Statistical analysis}

NCSS (Number Cruncher Statistical System) 2007 (Kaysville, Utah, USA) program was used for 


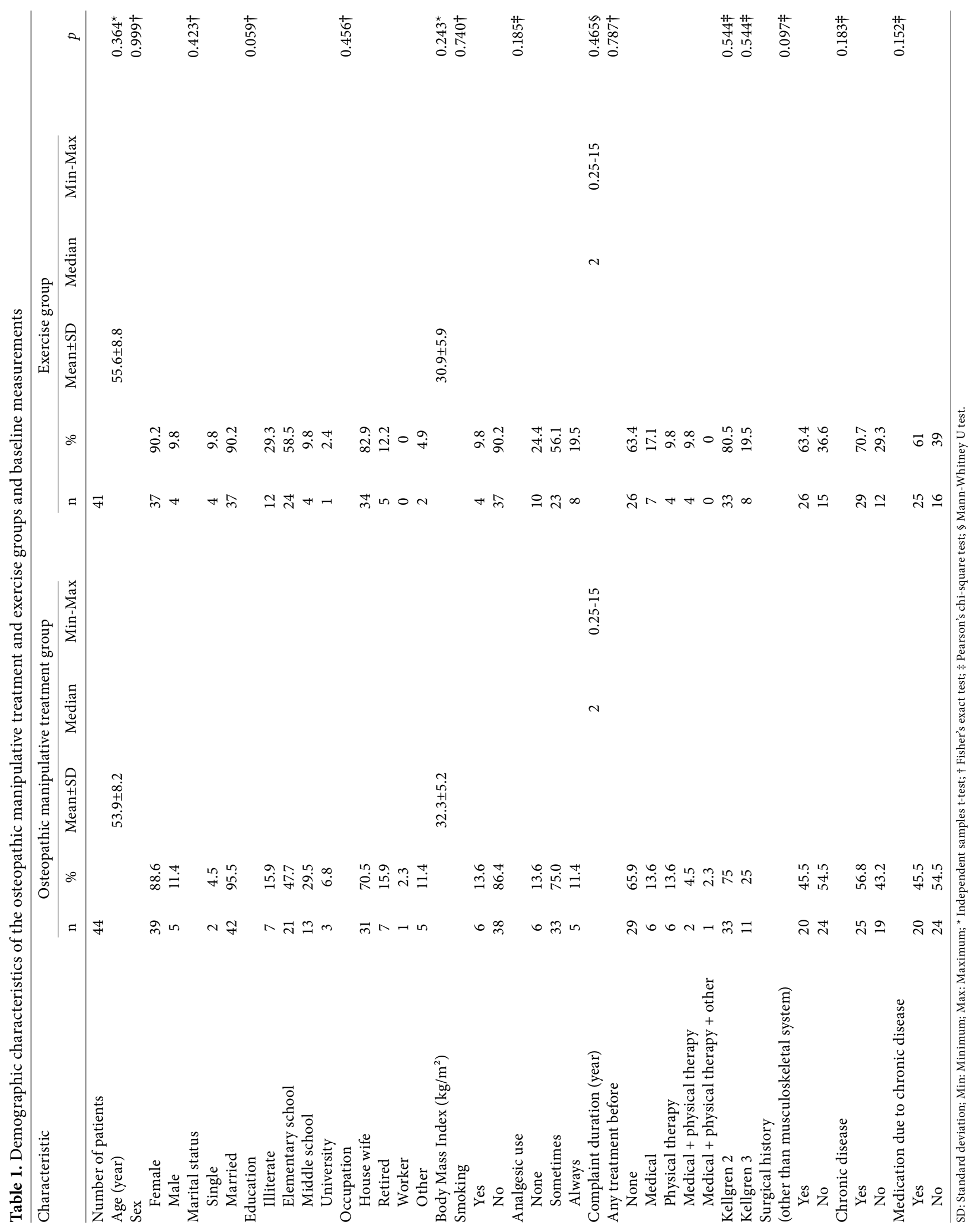




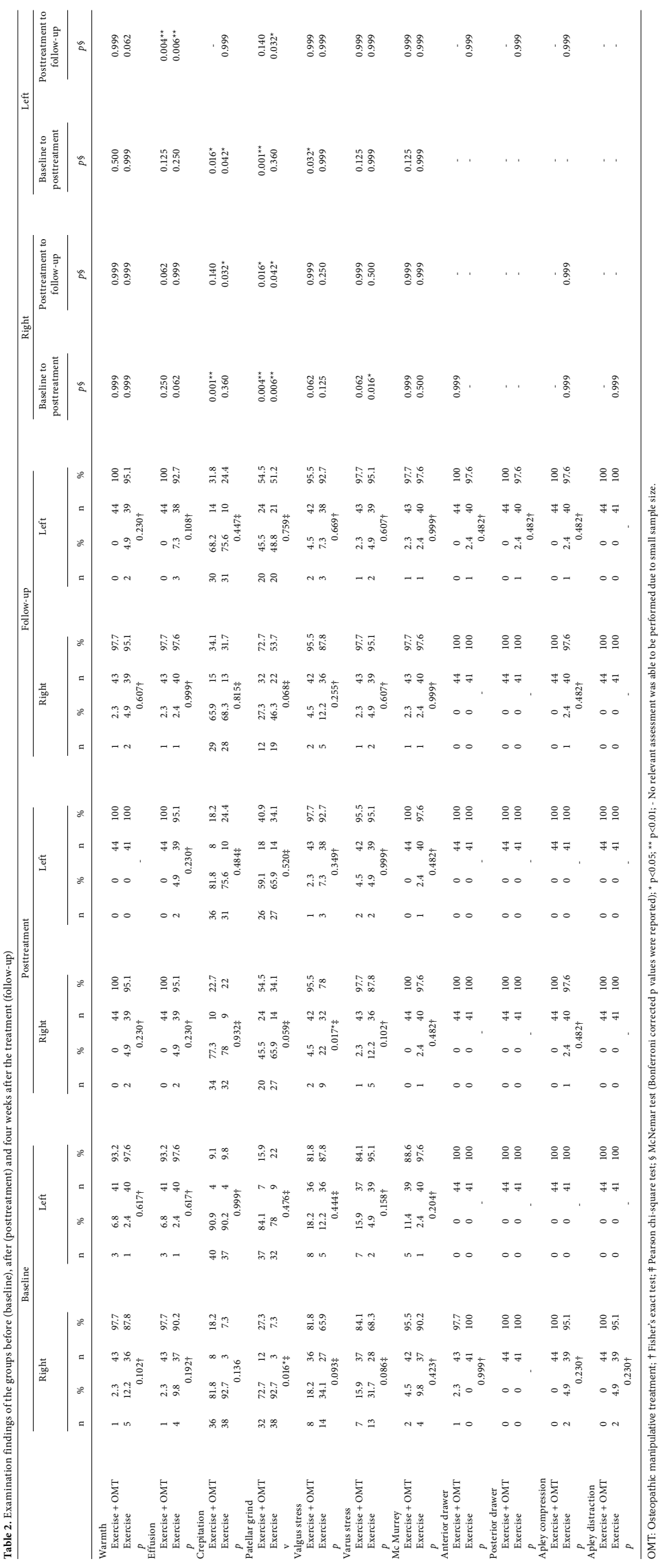




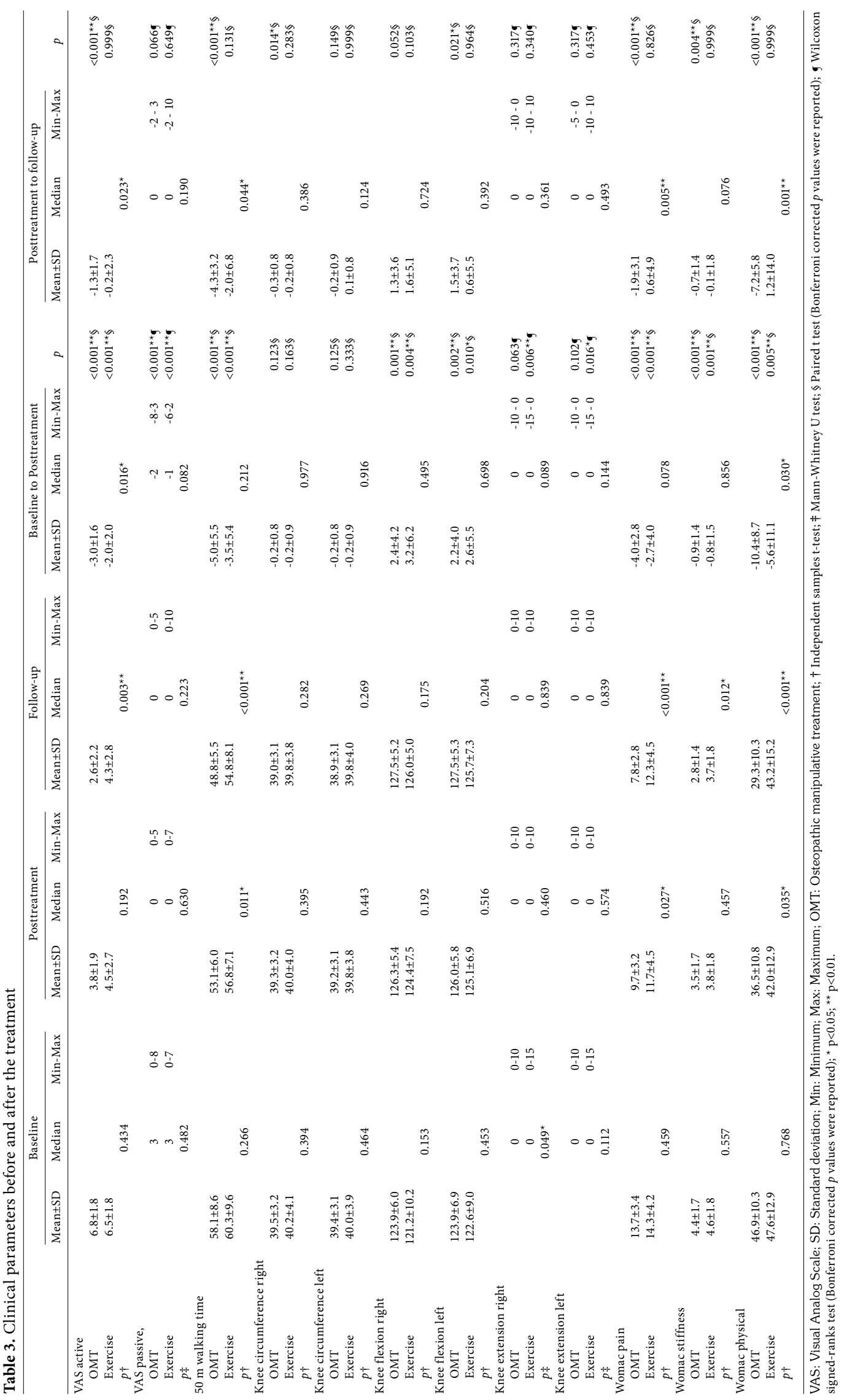


statistical analysis. Definitive were expressed in mean and standard deviation (SD), median (min-max), frequency, and ratio were used for defining the data. The convenience of quantitative variables to normal distribution was evaluated by Shapiro-Wilk test and graphical evaluations. Independent samples t-test was used for intergroup comparison of the normally distributed variables, and Mann-Whitney $\mathrm{U}$ test was used for intergroup comparison of the nonnormally distributed qualitative variables. We used paired t-test for evaluation of intra-group normally distributed variables, and Wilcoxon signed-ranks test for evaluation of intra-group non-normally distributed qualitative variables. Comparison of qualitative data was evaluated by Pearson chi-square test, Fisher's exact test, and McNemar test. An alpha level of $=0.05$ and a $p$ value of $<0.05$ were considered statistically significant.

\section{RESULTS}

Demographics of the patients included in the study are shown in Table 1. No statistically significant difference was found between the groups in terms of demographic characteristics ( $p>0.05)$.

In inter-group distribution, patients were homogeneous in laboratory tests and knee radiographs according to the radiological staging scale by Kellgren and Lawrence, and no statistically significant difference was observed between the groups ( $p>0.05$ ).

Physical examination findings (warmth, effusion, crepitation, patellar grind test, valgus stress, varus stress, McMurrey, anterior drawer, posterior drawer, Apley compression, Apley distraction, knee circumference measurement and range of motion) of the groups before, after and four weeks after the treatment are presented in Table 2. While there were no significant differences between the groups before the treatment $(p>0.05)$, findings after the treatment showed statistically significant differences $(p<0.05)$.

Clinical evaluation parameters (VAS, WOMAC, and $50-\mathrm{m}$ walking time) of the groups before, after and four weeks after the treatment are given in Table 3. While there were no significant differences between the groups before the treatment $(p>0.05)$, clinical parameters at the follow-up after the treatment showed statistically significant differences $(\mathrm{p}<0.05)$.

\section{DISCUSSION}

Osteoarthritis of the knee causes pain and functional limitation and, as it progresses, patients often enter a cycle of pain, inactivity, and weakness. Osteopathic manipulative treatment aims to stretch the soft tissues, detach tissue adhesions, increase blood flow and lymphatic drainage, decrease edema, improve range of motion, and lower the pain. Our findings revealed that OMT and exercise treatment together improves function and reliefs pain more than exercise treatment alone.

Muscle strengthening and aerobic exercises are found to be effective in reducing pain and improving physical function in patients with mild to moderate knee $\mathrm{OA}^{\left[{ }^{[11,12]}\right.}$ As we review the literature, we found that the combination of OMT and exercise was shown to reduce the need for total knee replacement and steroid injections. ${ }^{[13,14]}$ Moss et al. ${ }^{[15]}$ reported greater improvements in pain or stiffness in the OMT group, compared to the control group. Deyle et al. ${ }^{[13]}$ suggested that a combination of manual physical therapy and supervised exercise provided functional benefits in knee OA patients and it might delay or prevent the need for surgical intervention. Abbott et al. ${ }^{[16]}$ demonstrated that in OA patients with knee pain, manual physiotherapy prevailed standard care. The benefits were kept up to one year. Similarly, we demonstrated the functional benefits as well as pain relief and improvements in physical findings with exercise + OMT in this study.

On the other hand, Wang et al. ${ }^{[17]}$ reported equal results between manipulation and exercise versus manipulation alone in their clinical study. This study supports our results in a way, as they showed the clinical effect of manipulation on knee OA. We compared exercise and manipulation with exercise alone in our study. We also found a study ${ }^{[18]}$ in the literature review, which investigated the use of OMT in post-arthroplasty patients that reported OMT was not effective than a sham therapy in the post-surgical cases. However, the authors reported that the therapists in this study were studying osteopathy and they did not complete their education yet. Postoperative effects of OMT can be studied in the future, although currently we do not have sufficient reports and knowledge. Furthermore, Ebert et al. ${ }^{[19]}$ showed that manual lymphatic drainage techniques aid in the early postoperative stages after total knee arthroplasty and improve active knee flexion for up to six weeks postoperatively.

Manipulation is known with the high velocity, low amplitude manual thrusting procedures aiming to increase the motion of the joints. The results of manipulation-induced joint mobility consists decreased pain, decreased muscle hyperactivity, increased 
reflexes in the autonomic system, improvement in joint proprioception and, collectively, an increase in joint mobility. ${ }^{[20-22]}$ These effects compose the purpose of manipulation/manual therapy to the peripheral joints in clinical circumstances of acute or chronic (i.e., repetitive) strain and sprain of these joints as well as of chronic arthritic degeneration. ${ }^{[23]}$

In conclusion, OMT is a particular treatment used by osteopathic physicians to complement conventional treatment of musculoskeletal disorders. There are many studies showing the value of manual therapy in treating knee pain and osteoarthritis. Besides, expert opinions support that osteopathic manipulative treatment of the knee is useful in relieving knee pain. Therefore, it is assumed that OMT would provide similar benefits to those derived from studies reviewing other manual therapies.

\section{Declaration of conflicting interests}

The authors declared no conflicts of interest with respect to the authorship and/or publication of this article.

\section{Funding}

The authors received no financial support for the research and/or authorship of this article.

\section{REFERENCES}

1. Centers for Disease Control and Prevention (CDC). National and state medical expenditures and lost earnings attributable to arthritis and other rheumatic conditions--United States, 2003. MMWR Morb Mortal Wkly Rep 2007;56:4-7.

2. Elders MJ. The increasing impact of arthritis on public health. J Rheumatol Suppl 2000;60:6-8.

3. Kim RH, Springer BD, Douglas DA. Knee reconstruction and replacement. In: Flynn F, editor. Orthopaedic Knowledge Update. Rosemont, IL: American Academy of Orthopaedic Surgeons; 2011. p. 469-75.

4. Lo KS, Kuchera ML, Preston SC, Jackson RW. Osteopathic manipulative treatment in fibromyalgia syndrome. J Am Osteopath Assoc 1992;92:1177.

5. Enneking WF, Horowitz M. The intra-articular effects of immobilization on the human knee. J Bone Joint Surg Am 1972;54:973-85.

6. Rubin BR. Rheumatology. In: Ward RC, editor. Foundations for Osteopathic Medicine. Baltimore: Williams \& Wilkins; 1997. p. 459-66.

7. Rubin BR, Gamber RG, Cortez CA, Wright TJ, Shores J, Davis G. Treatment options in fibromyalgia syndrome. J Am Osteopath Assoc 1990;90:844-5.
8. Kellgren JH, Lawrence JS. Radiological assessment of osteoarthrosis. Ann Rheum Dis 1957;16:494-502.

9. Bellamy N. WOMAC Osteoarthritis Index. A user's guide. University of Western Ontario, London,. Ontario, Canada, 1995.

10. Available from: www.randomiser.org

11. Bennell KL, Hinman RS. A review of the clinical evidence for exercise in osteoarthritis of the hip and knee. J Sci Med Sport 2011;14:4-9.

12. Iwamoto J, Sato Y, Takeda T, Matsumoto H. Effectiveness of exercise for osteoarthritis of the knee: A review of the literature. World J Orthop 2011;2:37-42.

13. Deyle GD, Henderson NE, Matekel RL, Ryder MG, Garber $\mathrm{MB}$, Allison SC. Effectiveness of manual physical therapy and exercise in osteoarthritis of the knee. A randomized, controlled trial. Ann Intern Med 2000;132:173-81.

14. Iudica AC. Can a program of manual physical therapy and supervised exercise improve the symptoms of osteoarthritis of the knee? J Fam Pract 2000;49:466-7.

15. Moss P, Sluka K, Wright A. The initial effects of knee joint mobilization on osteoarthritic hyperalgesia. Man Ther 2007;12:109-18.

16. Abbott JH, Robertson MC, Chapple C, Pinto D, Wright AA, Leon de la Barra S, et al. Manual therapy, exercise therapy, or both, in addition to usual care, for osteoarthritis of the hip or knee: a randomized controlled trial. 1: clinical effectiveness. Osteoarthritis Cartilage 2013;21:525-34.

17. Wang JL, Chai $\mathrm{CH}, \mathrm{Xu}$ YM. Clinical observations on the effect of Tuigua manipulation combined with quadriceps exercise for the treatment of degenerative gonarthritis. Zhongguo Gu Shang 2008;21:887-9. [Abstract]

18. Licciardone JC, Stoll ST, Cardarelli KM, Gamber RG, Swift JN Jr, Winn WB. A randomized controlled trial of osteopathic manipulative treatment following knee or hip arthroplasty. J Am Osteopath Assoc 2004;104:193-202.

19. Ebert JR, Joss B, Jardine B, Wood DJ. Randomized trial investigating the efficacy of manual lymphatic drainage to improve early outcome after total knee arthroplasty. Arch Phys Med Rehabil 2013;94:2103-11.

20. Triano J. The theoretical basis for spinal manipulation. In: Haldeman S, editor. Principles and Practice of Chiropractic. New York: McGraw Hill; 2005. p. 361-82.

21. Millan, M, Leboeuf-Yde, C, Budgell, B, Descarreaux, M, Amorim, MA. The effect of spinal manipulative therapy on spinal range of motion: a systematic literature review. Chiropr Man Therap 2012;20:26.

22. Pickar JG, Bolton PS. Spinal manipulative therapy and somatosensory activation. J Electromyogr Kinesiol 2012;22:785-94.

23. Vernon H. Manipulation/manual therapy in the treatment of osteoarthritis. J Arthritis 2013;2:e107. 